\title{
Tracing the sites of obscured star formation in the Antennae galaxies with Herschel-PACS ${ }^{\star}$
}

\author{
U. Klaas ${ }^{1}$, M. Nielbock ${ }^{1}$, M. Haas ${ }^{2}$, O. Krause ${ }^{1}$, and J. Schreiber ${ }^{1}$ \\ 1 Max-Planck-Institut für Astronomie (MPIA), Königstuhl 17, 69117 Heidelberg, Germany \\ e-mail: klaas@mpia.de \\ 2 Astronomisches Institut, Ruhr-Universität Bochum (AIRUB), Universitätsstr. 150/NA7, 44801 Bochum, Germany
}

Received 31 March 2010 / Accepted 19 April 2010

\begin{abstract}
Aims. FIR imaging of interacting galaxies allows locating even hidden sites of star formation and measuring of the relative strength of nuclear and extra-nuclear star formation. We want to resolve the star-forming sites in the nearby system of the Antennae.

Methods. Thanks to the unprecedented sharpness and depth of the PACS camera onboard ESA's Herschel Space Observatory, it is possible for the first time to achieve a complete assessment of individual star-forming knots in the FIR with scan maps at 70, 100, and $160 \mu \mathrm{m}$. We used clump extraction photometry and SED diagnostics to derive the properties related to star-forming activity.

Results. The PACS 70, 100, and $160 \mu \mathrm{m}$ maps trace the knotty structure of the most recent star formation along an arc between the two nuclei in the overlap area. The resolution of the starburst knots and additional multi-wavelength data allow their individual star formation history and state to be analysed. In particular, the brightest knot in the mid-infrared (K1), east of the southern nucleus, exhibits the highest activity by far in terms of dust heating and star formation rate, efficiency, and density. With only $2 \mathrm{kpc}$ in diameter, this area has a 10-1000 $\mu \mathrm{m}$ luminosity, which is as high as that of our Milky Way. It shows the highest deficiency in radio emission in the radio-to-FIR luminosity ratio and a lack of X-ray emission, classifying it as a very young complex. The brightest 100 and $160 \mu \mathrm{m}$ emission region (K2), which is close to the collision front and consists of 3 knots, also shows a high star formation density and efficiency and lack of X-ray emission in its most obscured part, but an excess in the radio-to-FIR luminosity ratio. This suggests a young stage, too, but different conditions in its interstellar medium. Our results provide important checkpoints for numerical simulations of interacting galaxies when modelling the star formation and stellar feedback.
\end{abstract}

Key words. galaxies: interactions - galaxies: individual: Antennae - galaxies: ISM - galaxies: starburst galaxies: individual: ARP 244 - galaxies: individual: NGC 4038/39

\section{Introduction}

We have set up a photometric imaging programme of a sample of nearby interacting galaxies as part of the Herschel guaranteed time key project SHINING ${ }^{1}$ to trace and resolve the youngest star formation sites that are still embedded in dust, making them strong far-infrared (FIR) emitters. The Antennae (Arp 244, NGC 4038/NGC 4039) is a key object in this sample, since it is an interactive system, where strong extranuclear star formation was found previously in the so-called overlap area of the two disks between the two nuclei. Optical images show dark dust lanes interspersed with a few HII regions in this area (e.g. Whitmore \& Schweizer 1995). It houses several supergiant molecular cloud complexes (Stanford et al. 1990; Wilson et al. 2000; Gao et al. 2001; Schulz et al. 2007), which provide a large reservoir for bursts of star formation. Prominent 6 and $20 \mathrm{~cm}$ radio emission (Hummel \& van der Hulst 1986), which spatially matches the $\mathrm{CO}$ emisison peaks, suggests there are ongoing starbursts. Compressed magnetic fields are found

* Herschel is an ESA space observatory with science instruments provided by European-led Principal Investigator consortia and with important participation from NASA.

1 http://www .mpe.mpg.de/ir/Research/SHINING/ towards the northern edge indicating pre-starbursts (Chyzy \& Beck 2004).

Mid-infrared (MIR) observations have discovered embedded starbursts at the southeastern border of the overlap region (Vigroux et al. 1996; Mirabel et al. 1998; Wilson et al. 2000), while submm $850 \mu \mathrm{m}$ observations suggest a large amount of cooler dust in the northern overlap region, arguing in favour of relatively less powerful starbursts there (Haas et al. 2000; Zhu et al. 2003; Schulz et al. 2007).

In the case of buried starbursts, one expects to see the continuum re-emission of the hiding dust at mid- and far-infrared wavelengths. However, previous FIR space missions have not reached the spatial resolution required to separate the overlap region from the nuclei. Herschel-PACS now offers the capability of such spatially resolved observations for the first time.

\section{Observations and data reduction}

Arp 244 has been observed with the Photodetector Array Camera and Spectrograph (PACS) (Poglitsch et al. 2010) onboard the Herschel Space Observatory (Pilbratt et al. 2010) on Operational Day 208, between 2009-12-08T02:13:51Z and 2009-12-08T03:01:26Z as part of the science demonstration 


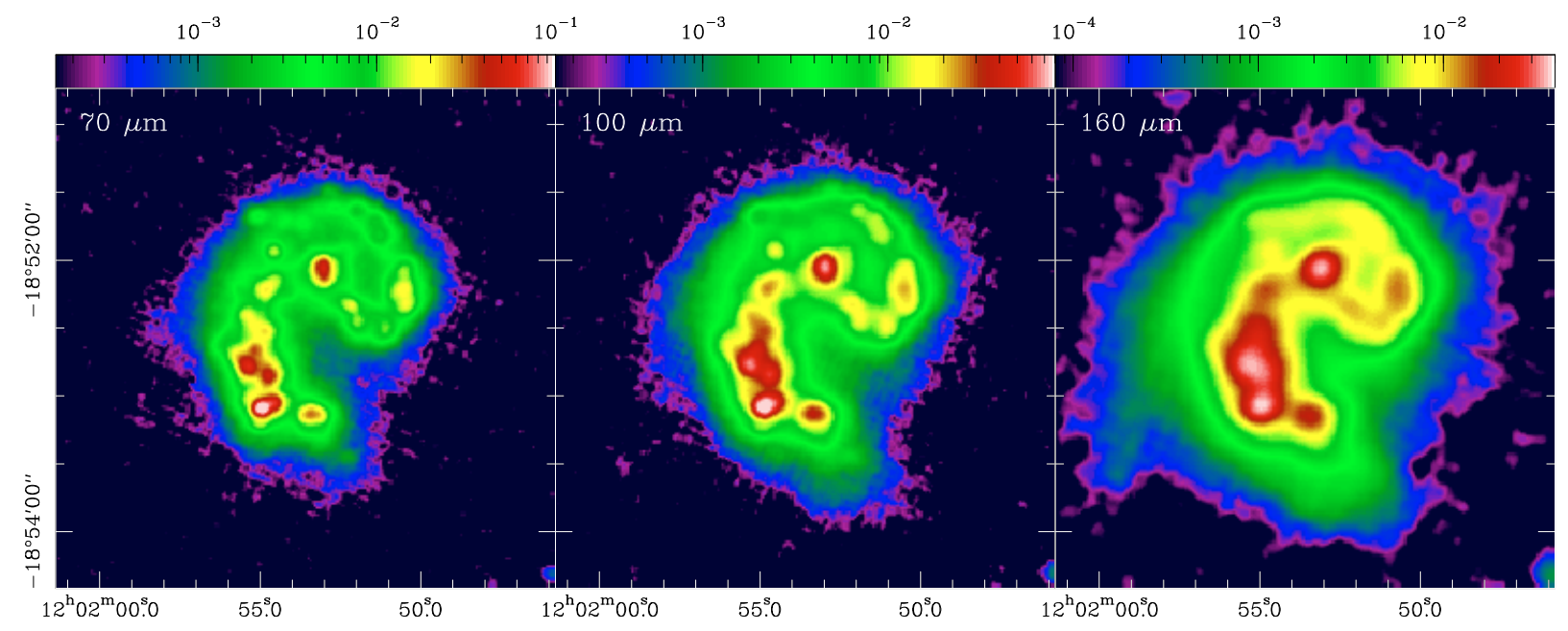

Fig. 1. PACS maps of the Antennae galaxies at 70, 100 and $160 \mu \mathrm{m}$. The look-up tables indicate the flux level in Jy/ם".

phase (SDP) using the three photometric bands centred on nominal wavelengths of 70, 100, and $160 \mu \mathrm{m}$. Four scan maps, 2 each in two orthogonal directions (45 and $135 \mathrm{deg}$ ), have been obtained with the medium scan speed of $20^{\prime \prime} \mathrm{s}^{-1}$. The design of the scans and cross-scans resulted in 8 scan legs of $6^{\prime}$ length and 10 scan legs of 4.5 length, respectively. The leg separation was $39^{\prime \prime}$.

The raw data were reduced with the Herschel interactive processing environment (HIPE) ${ }^{2}$ version 3.0, build 1134, running the standard steps of the PACS photometer pipeline (Poglitsch et al. 2010) and leading to level 1 calibrated data. Deglitching of the data was done via a moderate setting of the MMT deglitching task and a second-level deglitching on the intermediate level 2 products. A source mask was designed covering the emission area in the map that allowed the emission features to be excluded from the deglitching and the subsequent high-pass filtering of the data. It was applied with a median window of 41 data samples to remove the effect of bolometer signal drifts and the $1 / f$ noise. The flux correction factors provided by the PACS Instrument Control Centre (ICC) team have been applied. The FWHM of the PSF is 5.'5, 6.' 8 , and $11^{\prime \prime} 3$ in the 70, 100, and $160 \mu \mathrm{m}$ band, respectively. Astrometry of the PACS maps was corrected on three compact field sources.

For $15 \mu \mathrm{m}$ photometry, the ISOCAM map (Mirabel et al. 1998), provided to us by O. Laurent for our earlier publication (Haas et al. 2000), was used. For $24 \mu \mathrm{m}$ photometry, the SpitzerMIPS scan-map from programme 32 (P.I. G. Fazio) was reduced using the Data Analysis Tool (DAT; Gordon et al. 2005).

The photometry of the partially crowded emission knots of the $15 \mu \mathrm{m}$ ISOCAM, $24 \mu \mathrm{m}$ Spitzer-MIPS and 70, 100, and $160 \mu \mathrm{m}$ Herschel-PACS maps used the HIIphot package developed by Thilker (Thilker et al. 2000). The standard parameter settings were applied, except a maximum source size of $2000 \mathrm{pc}$ and a background annulus of $1500 \mathrm{pc}$ were used in order to have similar source borders, taking the variation in spatial resolution into account over the considered wavelength range. PSF FWHM and the termgrad array were adjusted according to the instrument/wavelength and the flux level of the individual maps.

\footnotetext{
${ }^{2}$ HIPE, as part of the Herschel common science system (HCSS), is a joint development by the Herschel Science Ground Segment Consortium, consisting of ESA, the NASA Herschel Science Center, and the HIFI, PACS, and SPIRE consortia. (See http://herschel. esac.esa.int/DpHipeContributors.shtml)
}

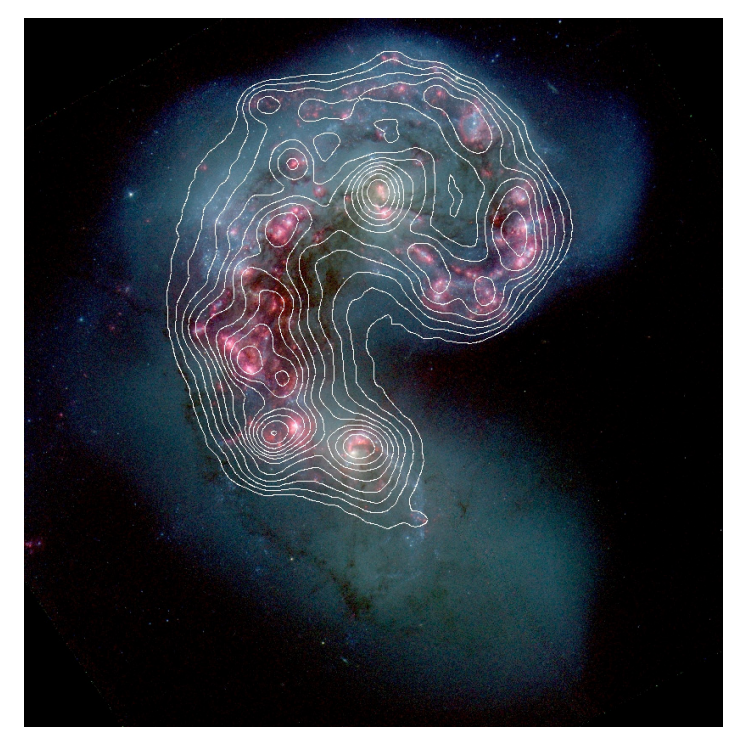

Fig. 2. PACS $100 \mu \mathrm{m}$ contours superimposed on a composite of HSTimages in the $F 435 W$ (blue), $F 550 M$ (green), and $F 658 N\left(\mathrm{H}_{\alpha}\right)$ filters.

\section{Results}

Figure 1 shows the Herschel-PACS maps at 70, 100, and $160 \mu \mathrm{m}$. The $160 \mu \mathrm{m}$ map reaches farthest out, since it has twice the exposure time of the blue $(70+100)$ bands. Its faintest contours cover the optical disks (cf. Fig. 2). There is some dust emission excess, where the optical image shows the deep cleft between the two disks. The $160 \mu \mathrm{m}$ image also indicates the base of the southern tidal arm where it shows a bulge in the optical, but there is no emission detected from the long thin tidal arms. The 70 and $100 \mu \mathrm{m}$ maps show a cut-off southwest of the NGC 4039 nucleus, and this very low flux level indicates a lack of heating sources and possibly also a depletion of dust in this area (Fig. 2).

The two nuclei and the chain of HII region complexes along the northwestern wound-up spiral arm of NGC 4038 are clearly resolved, as are several emission knots along an arc in the overlap region connecting the two nuclei (see Fig. 3 for the knot identification). Both nuclei are brighter than the HII regions in the spiral arm, but the brightest emission in all three bands comes from the overlap region. At $100 \mu \mathrm{m}$ the brightest emission (K1 east) lies at the southern edge of the overlap region. It coincides with the prominent 15 and $24 \mu \mathrm{m}$ emission peaks 


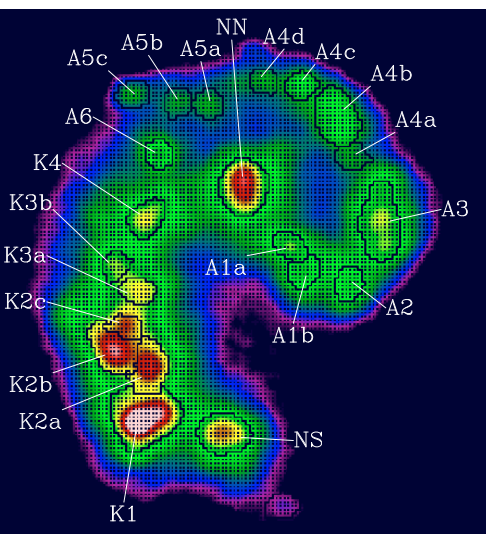

Fig. 3. GROW+BORDER map example of the HIIphot program (Thilker et al. 2000) on the PACS $70 \mu \mathrm{m}$ map illustrating the photometric apertures for the emission knots.

Table 1. Integral fluxes and fluxes of the emission knots from the ISOCAM $15 \mu \mathrm{m}$, Spitzer-MIPS $24 \mu \mathrm{m}$, and Herschel-PACS 70, 100, and $160 \mu \mathrm{m}$ maps.

\begin{tabular}{cccccc}
\hline \hline knot ID & \multicolumn{5}{c}{ fluxes (mJy) } \\
& $15 \mu \mathrm{m}$ & $24 \mu \mathrm{m}$ & $70 \mu \mathrm{m}$ & $100 \mu \mathrm{m}$ & $160 \mu \mathrm{m}$ \\
\hline integral & 1720 & 5960 & 67200 & 102000 & 95500 \\
\hline NS & 34.8 & 72.0 & 1726 & 1728 & 1489 \\
K1 & 341.5 & 1282 & 8144 & 8567 & 5030 \\
K2a & 61.0 & 159.9 & 2529 & 4004 & $6894^{2}$ \\
K2b+c & 81.2 & 251.9 & 3474 & 5999 & \\
K3a & 14.0 & 43.0 & 529.1 & 15491 & - \\
K3b & 7.8 & 21.9 & 316.1 & & - \\
K4 & 20.2 & 39.9 & 742.6 & 847.5 & 509.1 \\
NN & 84.7 & 260.7 & 3360 & 4904 & 4470 \\
A1a+b & 17.5 & 34.0 & 708.2 & 858.4 & 500.7 \\
A2 & 6.0 & 17.9 & 282.4 & 508.0 & - \\
A3 & 48.0 & 116.4 & 1695 & 1699 & 1353 \\
A4b & 12.1 & 20.5 & 698.6 & 693.8 & $744.6^{3}$ \\
A4c & 5.3 & 19.9 & 216.3 & 368.8 & \\
A4d & 3.8 & 5.9 & 81.6 & - & - \\
A5a & 3.3 & 5.7 & 64.9 & $340.2^{1}$ & $441.1^{2}$ \\
A5b & 3.7 & 4.5 & 66.5 & & \\
A5c & 2.7 & 4.9 & 110.5 & 237.9 & \\
A6 & 9.2 & 26.8 & 269.9 & 318.6 & - \\
\hline
\end{tabular}

Notes. The labelling of the knots follows Fig. 3. The photometric accuracy is estimated to be $\pm 20 \%$. ${ }^{(1)} \mathrm{a}+\mathrm{b} ;{ }^{(2)} \mathrm{a}+\mathrm{b}+;^{(c)} \mathrm{b}+\mathrm{c}$.

(Vigroux et al. 1996; Mirabel et al. 1998), which is located at the border of an optically bright HII region complex toward the dark dust lanes (Fig. 2). This region houses the supergiant molecular cloud complexes SGMC3-5 on the CO (1-0) map by Wilson et al. (2000). Two more bright FIR emission knots (K2a, K2b) are located north of $\mathrm{K} 1$ towards the optically obscured part of the overlap region (Fig. 2) and coincide with SGMC1 and SGMC2 on the CO (1-0) map by Wilson et al. (2000), peaks number 3 and 4 on the radio maps by Hummel \& van der Hulst (1986) and knot 2 of the $850 \mu \mathrm{m}$ map by Haas et al. (2000). Knots K2a and $\mathrm{K} 2 \mathrm{~b}$ together become the brightest area in the $160 \mu \mathrm{m}$ map.

Table 1 presents the HIIphot (Thilker et al. 2000) photometry of the whole system and the individual emission knots as labelled in Fig. 3. The integral photometry is very consistent with IRAS (Sanders et al. 2003) and ISOPHOT (Klaas et al. 1997) photometry. It is obvious that knots $\mathrm{K} 1$ and $\mathrm{K} 2$ by far exceed the brightness of the two nuclei NS(4039) and NN(4038), also
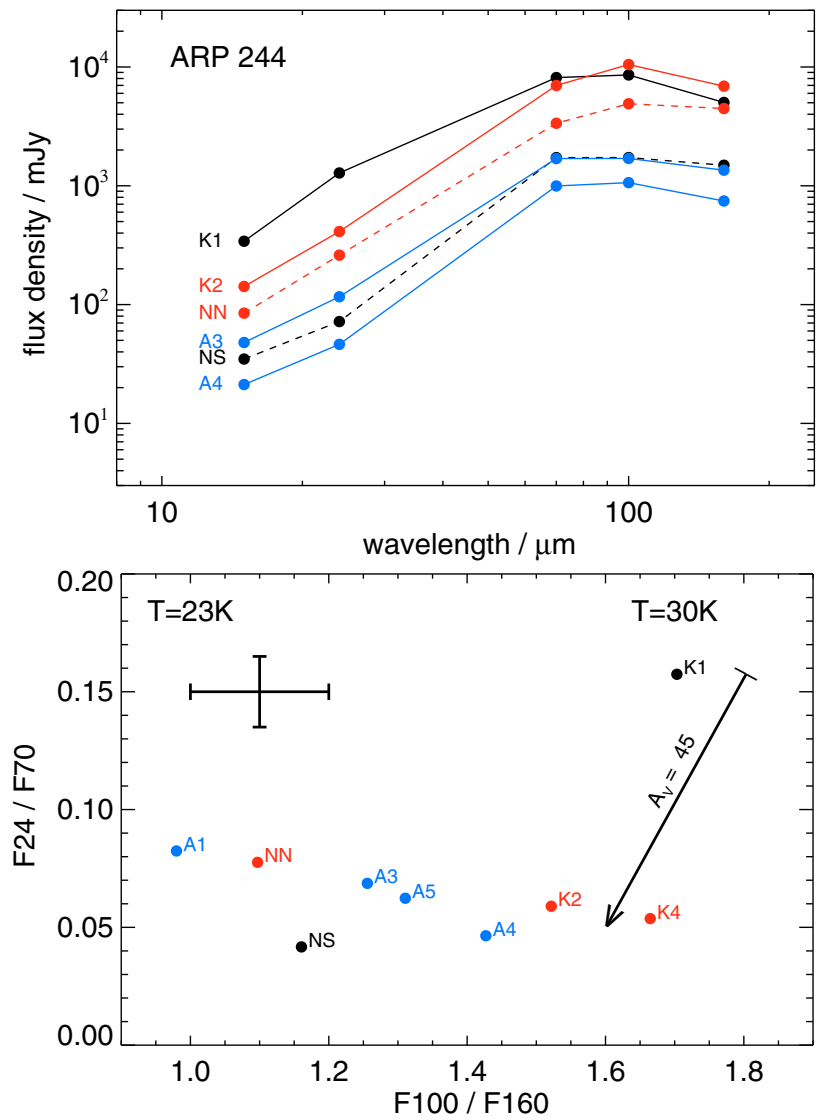

Fig. 4. Top: inter-comparison of the SEDs of the emission knots. Bottom: 2 colour diagram of the emission knots. Uncertainties due to the photometric accuracies are indicated by the cross. The 100/160 colour temperature range corresponding to a modified $\mathrm{BB}$ of shape $v^{2} \times \mathrm{B}(v, \mathrm{~T})$ and the extinction vector for $A_{\mathrm{V}}=45 \mathrm{mag}$ are indicated. The extinction curve of Mathis et al. (1983) has been used.

in the FIR. They are about 10 times brighter than the knots on the spiral arm.

In Fig. 4 we compare the MIR and FIR photometric properties of the most prominent and a few selected, less bright knots. All SEDs peak between 70 and $100 \mu \mathrm{m}$, with the exception of $\mathrm{NN}$, which is among the coldest emission knots. K1 clearly dominates the spectrum up to $70 \mu \mathrm{m}$, but for the longer wavelengths, it is slightly overtaken by the $\mathrm{K} 2$ area. From the colour-colour diagram in Fig. 4 it becomes obvious that the overlap area houses the most active heating sources. $\mathrm{K} 1, \mathrm{~K} 2$, and $\mathrm{K} 4$ all have dust colour temperatures above $27 \mathrm{~K}$, while the arm regions and the two nuclei are around $24 \mathrm{~K}$. K1 sticks out owing to its $24 \mu \mathrm{m}$ excess. It is remarkable that $\mathrm{K} 1$ gets an SED comparable to K2 when reddened with a visual extinction of $A_{\mathrm{V}}=45 \mathrm{mag}$. However, none of the other knots including the ones from the spiral arm reaches such a high 24/70 ratio as K1.

In Table 2 we have compiled important physical parameters of the emission knots: Size, corresponding to $n_{\text {pix }}$ of the HIIphot aperture (Col. 2), far-infrared luminosity, derived from multi-component modified BB fits to the photometry of Table 1 (Col. 3), colour temperature for a modified $\mathrm{BB}$ with $\beta=2$ (Col. 4), dust mass, as described in Wilke et al. (2004) (Col. 5), molecular gas mass, adding up all GMCs listed by Wilson et al. (2003) in the HIIphot aperture and re-scaling the distance (Col. 6), star formation rate and star formation density 
Table 2. Derived properties of the FIR emission knots.

\begin{tabular}{|c|c|c|c|c|c|c|c|c|c|c|}
\hline knot ID & $\begin{array}{c}\text { Size } \\
\left(\mathrm{kpc}^{2}\right)\end{array}$ & $\begin{array}{c}L_{\text {fir }} \\
\left(10^{9} L_{\odot}\right)\end{array}$ & $\begin{array}{l}T_{100 / 160} \\
(\mathrm{~K})\end{array}$ & $\begin{array}{c}M_{\text {dust }} \\
\left(10^{6} M_{\odot}\right)\end{array}$ & $\begin{array}{c}M_{\mathrm{H}_{2}} \\
\left(10^{8} M_{\odot}\right)\end{array}$ & $\begin{array}{c}\mathrm{SFR} \\
\left(M_{\odot} / \mathrm{yr}\right)\end{array}$ & $\begin{array}{c}\log \sum \mathrm{SFR} \\
\left(M_{\odot} \mathrm{yr}^{-1} \mathrm{kpc}^{-2}\right)\end{array}$ & $\begin{array}{c}\text { SFE } \\
\left(L_{\odot} M_{\odot}^{-1}\right)\end{array}$ & $\begin{array}{l}F_{6 \mathrm{~cm}} \\
(\mathrm{mJy})\end{array}$ & $\log \left(F_{6 \mathrm{~cm}} / F_{70 \mu \mathrm{m}}\right)$ \\
\hline integral & & 128.6 & 23.4 & 296.2 & 118.8 & 22.2 & & & 195 & -2.54 \\
\hline NS & 1.67 & 2.9 & 24.4 & 7.56 & 11.4 & 0.50 & -0.55 & 2.53 & 8 & -2.33 \\
\hline K1 & 4.68 & 16.1 & 30.0 & 3.63 & 17.0 & 2.78 & -0.23 & 9.47 & 16 & -2.71 \\
\hline K2a & 1.99 & 4.6 & 27.5 & 1.32 & 13.8 & 0.79 & -0.40 & 3.33 & 16 & -2.20 \\
\hline $\mathrm{K} 2 \mathrm{~b}+\mathrm{c}$ & 4.61 & 6.2 & 27.3 & 2.00 & 21.6 & 1.08 & -0.63 & 2.88 & 9 & -2.59 \\
\hline K4 & 1.50 & 1.1 & 29.5 & 0.20 & - & 0.19 & -0.90 & - & (2) & $(-2.57)$ \\
\hline NN & 3.22 & 6.8 & 23.7 & 4.79 & 31.0 & 1.17 & -0.44 & 2.19 & 12 & -2.45 \\
\hline A3 & 2.69 & 2.9 & 25.4 & 0.24 & 3.3 & 0.50 & -0.73 & 8.78 & 10 & -2.23 \\
\hline
\end{tabular}

Notes. For the meaning of the columns, see text. Values in parentheses are uncertain.

according to Kennicutt (1998) (Cols. 7 \& 8), star formation efficiency as the ratio of far-infrared luminosity and molecular gas mass (Col. 9), $6 \mathrm{~cm}$ radio flux from Hummel \& van der Hulst (1986) (Col. 10), and radio to far-infrared flux ratio according to de Jong et al. (1985) (Col. 11). The luminosity distance $D=28.4 \mathrm{Mpc}$ (from NED) was used for all distance-dependent measures.

\section{Discussion}

The Antennae is an interacting luminous $\left(10^{11} L_{\odot}\right)$ IR system close to the 2 nd encounter when the two galaxy disks will start to merge into a single system (e.g. Karl et al. 2008). The new FIR observations, along with the available radio and $\mathrm{CO}$ data, suggest that the gravitational forces have shuffled a huge amount of interstellar material along an arc connecting the two galaxy nuclei. Several kpc-sized, large ISM complexes house the most active star formation sites and evolve independently. With respect to their position on the optical image (Fig. 2), knots K2 and K3 appear to be located directly at the collision front of the two disks embedded in dense dust lanes but already associated with bright star clusters. Knot K1 is located more in the disk of NGC4039, while knot K4 is located in the disk of NGC 4038.

Knot K4 is a star formation area burning on a relatively low level. No CO emission is detected and there is no noticeable dust lane, so that it appears to have consumed its fuel and will start to extinguish soon.

Knot K2 reaches $2 / 3$ of the luminosity of $\mathrm{K} 1$, however the derived star formation densities and efficiencies are considerably lower than for K1. The spatially resolved SEDs obtained from the PACS maps clarify that it is more active than was suggested by Haas et al. (2000) on the basis of the ISO and the 450 and $850 \mu \mathrm{m}$ SCUBA maps. If $\mathrm{K} 2$ suffered from higher obscuration than $\mathrm{K} 1$ (see Fig. 4), the intrinsic difference between K2 and $\mathrm{K} 1$ would become smaller. There seems, however, to be a dichotomy between $\mathrm{K} 2 \mathrm{a}$ and $\mathrm{K} 2 \mathrm{~b}+\mathrm{c}$. While the latter appears to have a normal radio-to-FIR ratio, K2a shows an excess in radio emission, as already noted by Chyzy \& Beck (2004). It is very inconspicuous in the optical and is lacking X-ray emission (see Fabbiano et al. 2001, Fig. 9). K2a might therefore be in a younger evolutionary stage than $\mathrm{K} 2 \mathrm{~b}+\mathrm{c}$.

Knot K1 is currently the most active area by far. This is manifested by the highest individual luminosity, which itself is as high as the Milky Way luminosity but concentrated in an area of $2 \mathrm{kpc}$ diameter. It exhibits the highest star formation rate and efficiency, and the highest star formation density that consequently raises dust temperatures to a maximum. The high density of hot heating sources can explain the very high MIR emission. Its radio-to-FIR luminosity ratio deviates from the average in that there is a lack of radio emission, and at the peak position, there is no X-ray emission either (see Fabbiano et al. 2001, Fig. 9). These findings classify it as a very young complex.

\section{Conclusions}

New Herschel-PACS scan maps of the Antennae at 70, 100, and $160 \mu \mathrm{m}$ provide a high spatial resolution complement to earlier MIR maps (ISOCAM, Spitzer-MIPS), high-resolution CO (1-0), and radio $6 \mathrm{~cm}$ maps and thus allow detailed study of the star formation state in individual emission knots.

We confirm that the highest star formation activity takes place in the overlap area concentrated in the two emission complexes K1 and K2. Our star formation diagnostics strongly indicates that these knots differ in evolutionary stage.

Acknowledgements. PACS has been developed by a consortium of institutes led by MPE (Germany) and including UVIE (Austria); KUL, CSL, IMEC (Belgium); CEA, OAMP (France); MPIA (Germany); IFSI, OAP/AOT, OAA/CAISMI, LENS, SISSA (Italy); IAC (Spain). This development has been supported by the funding agencies BMVIT (Austria), ESA-PRODEX (Belgium), CEA/CNES (France), DLR (Germany), ASI (Italy), and CICYT/MCYT (Spain). M.H. was supported by the Nordrhein-Westfälische Akademie der Wissenschaften und der Künste. This research made use of the NASA/IPAC Extragalactic Database (NED). HST images were retrieved from the Canadian Astronomical Data Centre (CADC) Virtual Observatory via the Aladin tool. We thank the referee for constructive comments.

\section{References}

Chyzy, K., \& Beck, R. 2004, A\&A, 417, 541

de Jong, T., Klein, U., Wielebinski, R., \& Wunderlich, E. 1985, A\&A, 147, L6 Fabbiano, G., Zezas, A., \& Murray, S. 2001, ApJ, 554, 1035

Gao, Y., Lo, K., Lee, S.-W., \& Lee, T.-H. 2001, ApJ, 548, 172

Gordon, K., Rieke, G., Engelbracht, C., et al. 2005, PASP, 117, 503

Haas, M., Klaas, U., Coulson, I., Thommes, E., \& Xu, C. 2000, A\&A, 356, L83 Hummel, E., \& van der Hulst, J. 1986, A\&A, 155, 151

Karl, S., Naab, T., Johansson, P., Theis, C., \& Boily, C. 2008, AN, 329, 1042 Kennicutt, R. 1998, ApJ, 498, 541

Klaas, U., Haas, M., Heinrichsen, I., \& Schulz, B. 1997, A\&A, 325, L21

Mathis, J., Mezger, P., \& Panagia, N. 1983, A\&A, 128, 212

Mirabel, I., Vigroux, L., Charmandaris, V., et al. 1998, A\&A, 333, L1

Pilbratt, G. L., Riedinger, J. R., Passvogel, T., et al. 2010, A\&A, 518, L1 Poglitsch, A., Waelkens, C., Geis, N., et al. 2010, A\&A, 518, L2

Sanders, D., Mazzarella, J., Kim, D.-C., J.A., S., \& B. T., S. 2003, AJ, 126, 1670

Schulz, A., Henkel, C., Muders, D., et al. R. 2007, A\&A, 466, 467

Stanford, S., Sargent, A., Sanders, D., \& N. Z., S. 1990, AJ, 109, 960

Thilker, D., Braun, R., \& Walterbos, R. 2000, AJ, 120, 3070

Vigroux, L., Mirabel, F., Altieri, B., et al. 1996, A\&A, 315, L93

Whitmore, B., \& Schweizer, F. 1995, AJ, 109, 960

Wilke, K., Klaas, U., Lemke, D., et al. 2004, A\&A, 414, 69

Wilson, C., Scoville, N., Madden, S., \& Charmandaris, V. 2000, ApJ, 542, 120 Wilson, C., Scoville, N., Madden, S., \& Charmandaris, V. 2003, ApJ, 599, 1049 Zhu, M., Seaquist, E., \& Kuno, N. 2003, ApJ, 588, 243 